\title{
The Accuracy Rate of Holt-Winters Model with Particle Swarm Optimization in Forecasting Exchange Rates
}

\author{
Riz Rupert L. Ortiz* \\ Northwest Samar State University, Philippines. \\ * Corresponding author. Tel.: 055-2096753; email: rizortiz@gmail.com \\ Manuscript submitted July 3, 2015; accepted September 6, 2015. \\ doi: 10.17706/jcp.11.3.216-224
}

\begin{abstract}
Exchange rates forecasting is a crucial and challenging task. Accurate forecasting of the imminent movements of exchange rates is very important in investments, trade and economics. In this paper, an exponential smoothing using the Holt-Winters Model is used for forecasting exchange rates. Parameter search for the smoothing constants is done through computer simulation using Particle Swarm Optimization (PSO). Experiment results show that PSO is able to compute good values for the smoothing constants, producing forecasts with accuracy in determining the direction (rise or fall) of exchange rates. Furthermore, the computed Mean Absolute Deviation (MAD) and the Residual Standard Error (RSE) of the exchange rate forecasts from the actual observed data indicate that PSO can also be used to improve forecasting precision.
\end{abstract}

Key words: Simulation, forecasting, holt-winters model, particle swarm optimization.

\section{Introduction}

Exchange rate is an essential economic variable, and its movements can make significant impacts on the trading relationships between different economies, international capital flows and interest rates [1]. It is also one of the most important financial markets for investors. Consequently, accuracy in forecasting the upcoming movements of exchange rates translates to a huge practical value for foreign trade policy making, international investments and interest rate adjustments. However, generating accurate forecasts is very difficult because exchange rate is a highly nonlinear and non- stationary time series [2]. Previous studies have shown that using conventional methodology based on statistics and economics can hardly forecast the exchange rate [3], [4].

This study will use the Holt-Winters Model and will apply the Particle Swarm Optimization (PSO) to forecast exchange rates. The study aims to produce quite accurate predictions using some optimization method for the smoothing parameters of the Holt-Winters Model.

\section{Review of Related Literature}

Traditionally, the exchange rate prediction models are associated with certain classic financial models in forecasting. Many methodologies have been applied to exchange rates forecasting as revealed in the literature. Among the most extensively used forecasting techniques in business are exponential smoothing methods [5]. These include Single Exponential Smoothing Technique, Double Exponential Smoothing Technique, HW Simple Exponential Smoothing Technique, HW Multiplicative Exponential Smoothing Technique, HW Additive Exponential Smoothing Technique and ARIMA Models [6]. 
The latest developments in financial research offer a class of state space models that contain both ARIMA models and dynamic nonlinear statistical models wherein exponential smoothing methods are found to be optimal [7]. In the study of [8], the Holt-Winters method produced an extremely high forecasting accuracy. However, it is not easy to determine the optimal smoothing parameters for the Holt- Winters method. These parameters are usually approximated by minimizing the mean absolute deviations or mean squared errors. There are various heuristic algorithms that have been developed for approximating the smoothing constants. However, there are no empirical evidences that favor any particular method. Recently, optimization methods have been proposed in the estimation of starting values for these smoothing parameters [8], [9].

There are also various techniques for optimization search and parameter estimation. Some of the more popular techniques include Genetic Algorithm (GA), Simulated Annealing (SA), Artificial Neural Networks (ANN) and Particle Swarm Optimization (PSO). Some of these techniques have been applied to financial forecasting already. For instance, Nelly and Weller showed that GA is better than traditional time series model in predicting the fluctuation of exchange rates [10]. The research conducted by [11] compared ANN with traditional econometrics. They found that nonlinear mathematical models of multilayer perceptron, along with radial basis function neural networks, are able to provide more accurate forecasts than the traditional auto regressive moving average (ARMA) and ARMA generalized auto regressive conditional heteroskedasticity (ARMA-GARCH) linear models.

The Winters-PSOARMA model is a novel hybrid model used by [12] to forecast the opening price trend series in Shenzhen Stock Market. The model that takes advantages of the benefits of Holt-Winters, ARMA and PSO procedure is compared with the conventional Winter-ARMA model to forecast the series that has nonlinear and linear features. Accordingly, the time series is considered to be non-stationary. Results showed that their model is significantly more effective.

On the other hand, Natarajan and Periasamy used both PSO and back propagation (BPN) techniques to train a neural network; results showed that PSO is preferred over BPN. Those evidences showed that training the neural network by PSO technique gives more accurate results [13]. This study extends some of the previous studies by combining an existing double exponential smoothing method (Holt-Winters) and a parameter search algorithm (PSO).

The remainder of the paper is organized as follows: Section 3 explains the Holt-Winters and PSO techniques. Section 4 discusses the research methodology applied in this study. Section 5 presents the results and the accompanying analyses. The conclusions and suggestions for further directions are discussed in Section 6.

\section{Preliminaries}

\subsection{Holt-Winters Model}

The Holt-Winters Smoothing Algorithm uses weighted historical trending to predict the future values of an account. It is more accurate for accounts that tend to trend in one direction over time. The modified version of this algorithm looks at the financial data from past years and determines a value to place on the trend itself [14].

Holt-Winters Model is a double exponential smoothing method that is appropriate for series with a linear trend and no seasonal variations. It is an extension of simple exponential smoothing method that is originally designed for time series with no trend nor seasonal patterns. Basically, the Holt-Winters model contains two vital components; an exponentially smoothing constant $(E, \alpha)$ and a trend component $(T, \beta)$. The forecasted series is computed using the formula below: 


$$
F_{t+k}=E_{t}+k * T_{t}
$$

Here, $E$ and $T$ represent the intercept and slope respectively, and are computed recursively as follows:

$$
\begin{gathered}
E_{t}=\alpha *\left(Y_{t}\right)+(1-\alpha) *\left(\mathrm{E}_{t-1}+T_{t-1}\right) \\
T_{t}=\beta *\left(E_{t}-E_{t-1}\right)+(1-\beta) * T_{t-1}
\end{gathered}
$$

The symbols in the model are defined as follows:

$F_{t+k}=$ forecast value k periods from $t$

$Y_{t-1}=$ actual value for previous period $(t-1)$

$E_{t-1}=$ estimated value for previous period $(t-1)$

$T_{t-1}=$ trend value for previous period $(t-1)$

$\alpha=$ smoothing constant for estimates

$\beta=$ smoothing constant for trend

$k=$ number of periods

The coefficients $\alpha$ and $\beta$ are smoothing factors, each coming from the closed interval [0,1]. These coefficients are usually determined by minimizing the Residual Standard Error (RSE), Sum of Squared Errors (SSE), Mean of Squared Errors (MSE) or Mean Absolute Deviation (MAD). When $\alpha$ and $\beta$ have been selected, the smoothing equations are then employed to update the estimates of the constant and trend components. The updated estimates are used to compute forecasts of future time series values.

\subsection{Particle Swarm Optimization}

Particle swarm optimization (PSO) is a search algorithm that uses a population (i.e., swarm) of particles to explore a search space and then returns the particle that is said to be optimal [15]. Each particle represents a candidate solution to the optimization problem.

A particle of the swarm explores the search space based on a given mathematical formula using the particle's position and velocity. At each iteration, the particle's movement is guided by its pbest (the best solution achieved so far by the particle) and the swarm's gbest (the best solution achieved so far by all particles). Using these values, the particle updates its velocity $V[t, d]$ and position $X[t, d]$ at time t for each iteration and for each dimension (or coordinate) $d[16]$. In this study, the following update rules were used:

$$
\begin{gathered}
V[t, d]=U(-1,1) * c_{1}(p \operatorname{best}[d]-X[t-1, d])+U(-1,1) * c_{2}(g \operatorname{best}[d]-X[t-1, d]) \\
X[t, d]=X[t-1]+V[t, d]
\end{gathered}
$$

In the above equations, $U(-1,1)$ represents a randomly generated number from -1 to 1 coming from a uniform distribution. The coefficients $c_{1}$ and $c_{2}$ represent the user-defined parameters that affects the drive of the particles toward the pbest and gbest respectively.

A satisfactory solution is usually discovered by repeating the process and having several iterations. As the particles are guided towards the best known position of the entire swarm, PSO can converge to the optimal (or nearly optimal) point even without having to reach a large number of iterations. The PSO algorithm is shown in Fig. 1.

\section{Methodology}

This section presents the development process of optimizing Holt-Winters forecasting parameters using 
Particle Swarm Optimization (PSO).

\subsection{Implementation}

The PSO code was written in the JAVA programming language. Verification was made to ensure that the model was built properly. Specifically, testing and source code review was applied.

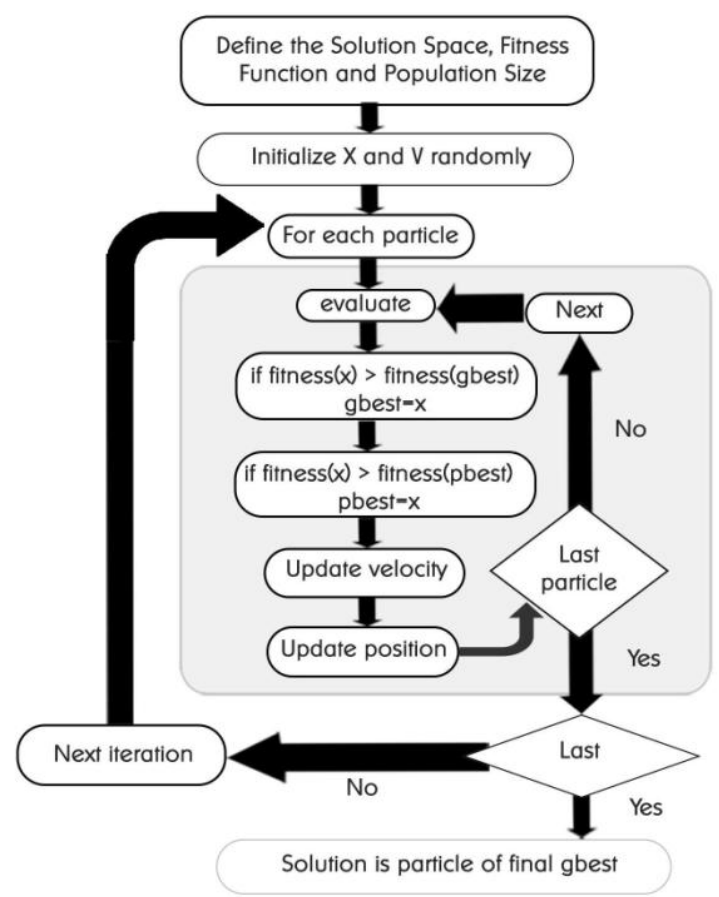

Fig. 1. Diagram showing the PSO Algorithm [17].

In this implemented PSO, each particle explores the search space to find the best smoothing constants $\alpha$ and $\beta$ for the Holt-Winters method. The optimal particle is the one that minimizes the difference between the actual data and the forecast made from the Holt-Winters method that use the particle's smoothing constant values. For this implementation, 1000 particles were used, and each particle underwent 1000 iterations. The values used for the PSO coefficients are as follows: $c_{1}=0.4$ and $c_{2}=0.4$. These constants corresponds to the weights of the drive towards personal and global bests respectively.

\subsection{Building the Holt-Winters Model}

Historical data was utilized in building the forecasting model. Specifically, Philippine exchange rates from year 2005 to 2015 were retrieved for this purpose. Three different currencies (Dollar, Euro and Yen) were used to produce the 3 datasets. It should be noted that the datasets come from actual published exchange rates, and are therefore considered clean (i.e., without noise).

A pre-test on the dataset to show that the time series has a linear trend was implemented using Zaitun Time Series application. It is a free and open source software designed for statistical analysis of time series data. It provides several statistics and graphical tools for time series modeling, forecasting and trend analysis. The following graphs (Figs. 2, 3 and 4) show the line plot for actual and predicted values of trend model for the three currencies. The trend analysis model using the software included around 126 observations for each dataset. The corresponding R and R-Square Adjusted values are as follows: 0.553787 and 0.985317 for Dollar-Peso; 0.602598 and 0.989768 for Euro-Peso; and 0.003979 and -0.546346 for Yen-Peso Exchange Rates. The low values for the Yen-Peso rates indicate that the linear trend assumption of the Holt-Winters model is not satisfied.

To establish the predictive ability of the model, each dataset was partitioned into 2 segments: Training 
Set and Test Set. The Training Set was used in the execution of PSO, in order to find the smoothing constants. The Test Set was used to evaluate the accuracy of the built Holt-Winters model as described in the next section.

Table 1 shows that for the Training Set, 84 months (January 2005 - December 2011) of data was used in generating the model containing the optimized smoothing constants of the Holt-Winters Model. Consequently, a total of 42 months were used for the Test Set (January 2012 - June of 2015).

Table 1. Historical Data of Exchange Rates

\begin{tabular}{|c|c|c|}
\hline Data Set & Period of Time & Number of Months \\
\hline Training Set & Jan 2005 - Dec 2011 & 84 months \\
\hline Test Set & Jan 2012 - June 2015 & 42 months \\
\hline
\end{tabular}

For this study, 1000 simulation runs were conducted in order to achieve a low variance for the results.

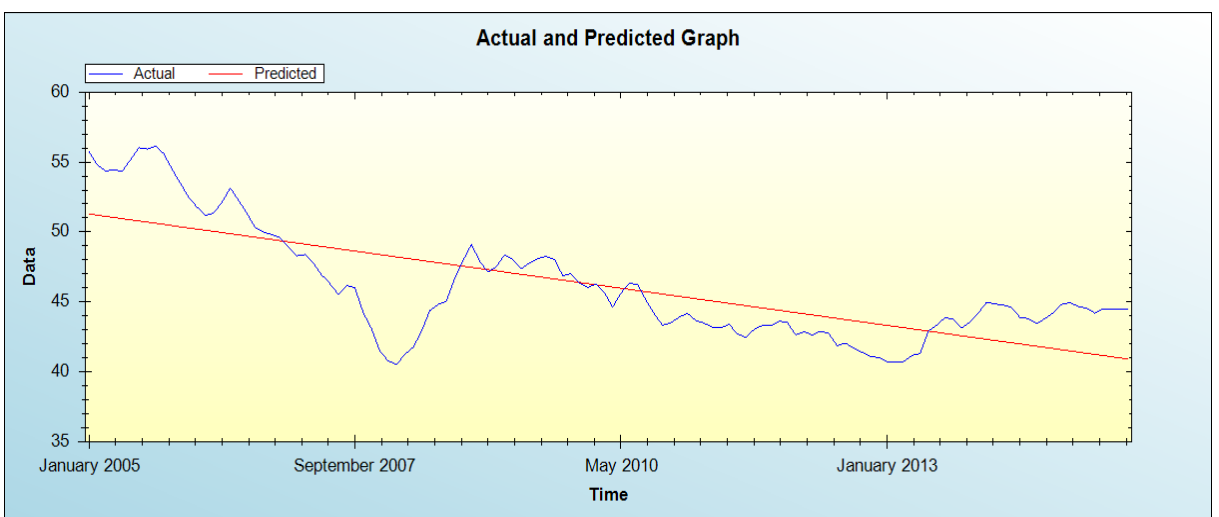

Fig. 2. Trend analysis for the dollar exchange rates.

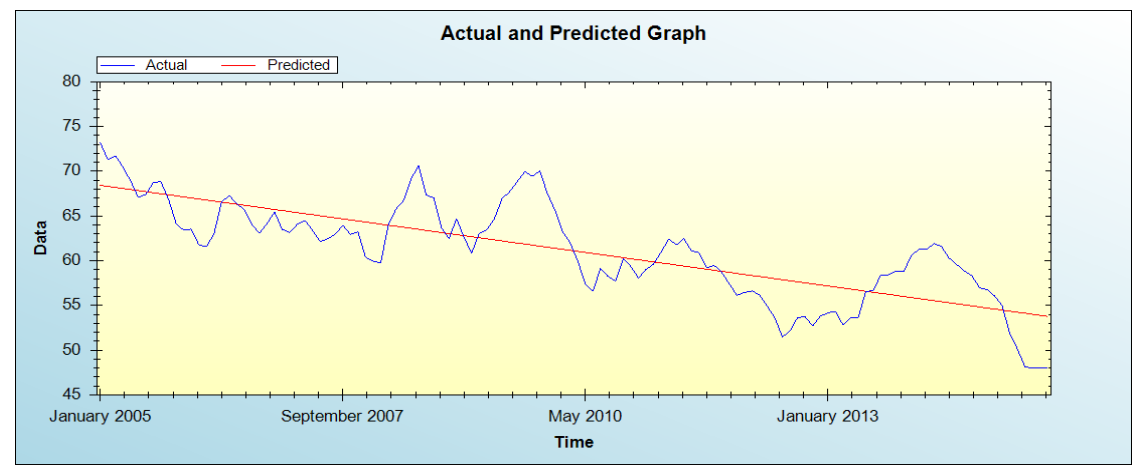

Fig. 3. Trend analysis for the euro exchange rates.

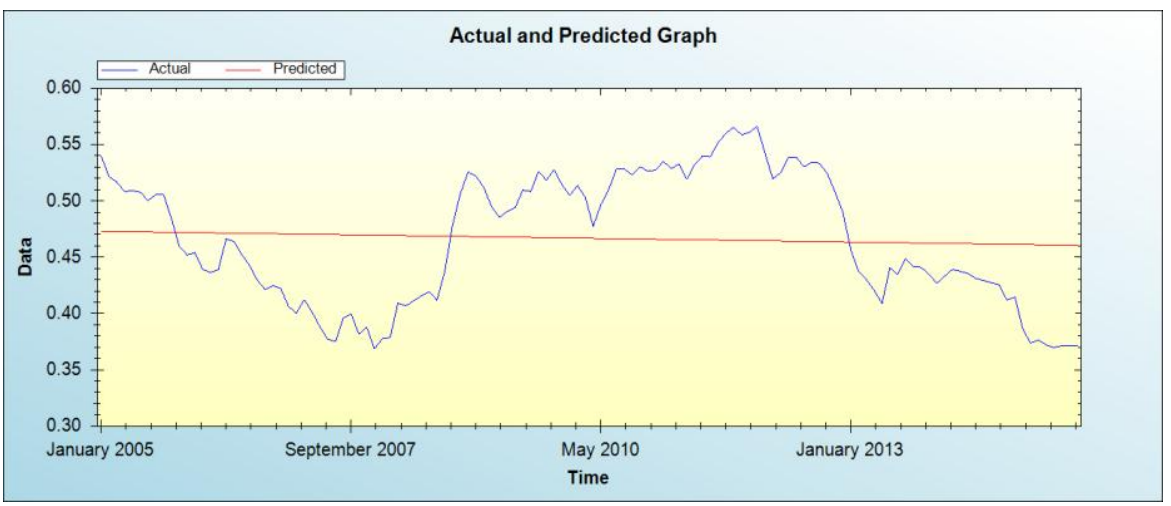

Fig. 4. Trend analysis for the yen exchange rates. 


\subsection{Model Evaluation}

The proposed model is finally evaluated using two types of measures. The first computes the accuracy of the direction forecast. If the observed data increases and the forecast value also increases, then the direction forecast is deemed correct. The accuracy rate for this is adapted from [18] and is defined as follows:

$$
\text { Accuracy _ Rate }=\frac{\text { Number_of_Correct_Direction__Forecasts }}{\text { Total_Number_of_Direction__Forecasts }}
$$

The second type of measure compares directly the forecast values with the actual data instead of just their directions. For this purpose, the following statistics were used in the measuring forecasting errors.

Mean Absolute Deviation (MAD)

$$
\mathrm{MAD}=\sum_{t=1}^{n} \frac{\left|Y_{t}-Y_{t}^{\prime}\right|}{n}
$$

Residual Standard Error (RSE)

$$
\mathrm{RSE}=\sqrt{\sum_{t=1}^{n} \frac{\left|Y_{t}-Y_{t}^{\prime}\right|^{2}}{n-1}}
$$

\section{Results and Analysis}

To validate the consistency and to test the accuracy rate of the forecast, the study considered three different currencies (Dollar, Euro and Yen). However, due to the poor results on the linear trend analysis generated from the pretesting of the dataset, the study opted to exclude the Peso-Yen Ex- change Rates. The historical data from the Training Set (Dollar and Euro) were used for training the model.

Table 2 shows the alpha and beta smoothing constants for each currency with their corresponding Mean Absolute Deviation (MAD) values. The smoothing coefficient values were derived using the PSO algorithm with 1000 particles and 1000 of iterations. The simulation then predicts exchange rates using the optimized smoothing constants of the Holt- Winter forecasting method.

Table 2. Alpha and Beta Smoothing Constants for the Holt-Winters Model Using the Training Set

\begin{tabular}{|c|c|c|}
\hline Currency & Alpha & Beta \\
\hline Dollar-Peso & 0.99342 & 0.85166 \\
\hline Euro-Peso & 0.98417 & 0.29141 \\
\hline
\end{tabular}

Table 3 shows the absolute error measures of the model (as used in two different currencies) for the Training Set. The smaller the values we get for MAD and RSE, the better is the model in terms of forecasting accuracy.

Table 3. Absolute Error Measures of the Model Using the Test Set

\begin{tabular}{|c|c|c|}
\hline Currency & MAD & RSE \\
\hline Dollar-Peso & 0.43169 & 0.66500 \\
\hline Euro-Peso & 1.04236 & 1.03334 \\
\hline
\end{tabular}

Table 4 shows the results of the performance of the model that was applied to the Test Set of the 
historical data. Accuracy rates of the different currencies involved in the study were computed and are shown in the table. The corresponding accuracy rates are, 52.38\% and 54.76\% for the Dollar and Euro respectively. There are lots of factors affecting the fluctuations of foreign exchange rates. Nevertheless, the optimized smoothing constants derived from PSO resulted in a forecasting model that performed quite well.

Table 4. Accuracy Rates of the Holt-Winters Forecasts

\begin{tabular}{|c|c|c|c|}
\hline Currency & Correct Forecast & Total Forecast & Accuracy Rate \\
\hline Dollar-Peso & 22 & 42 & 0.52381 \\
\hline Euro-Peso & 23 & 42 & 0.54762 \\
\hline
\end{tabular}

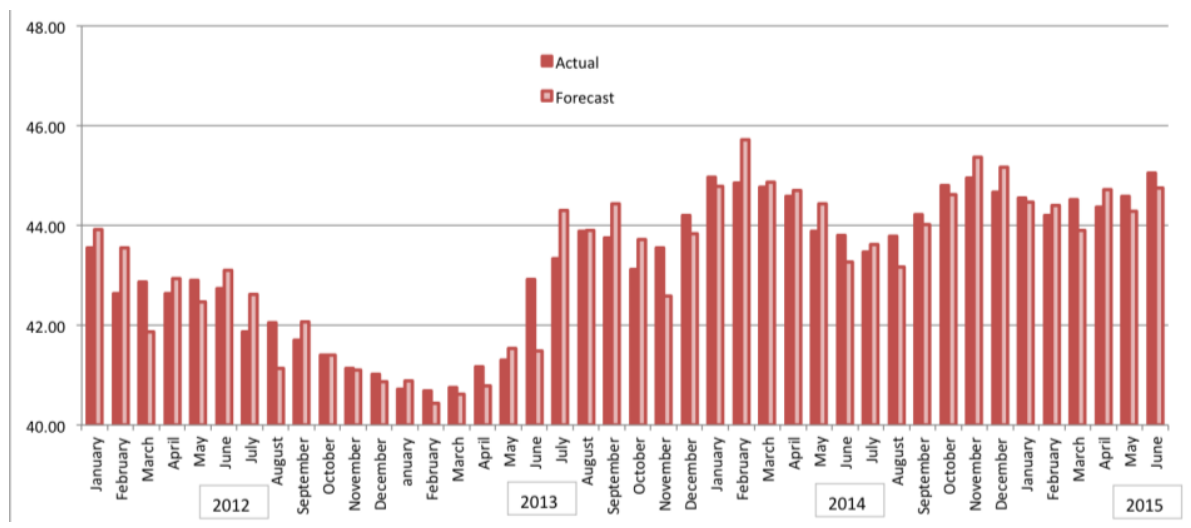

Fig. 5. Dollar-peso exchange rates.

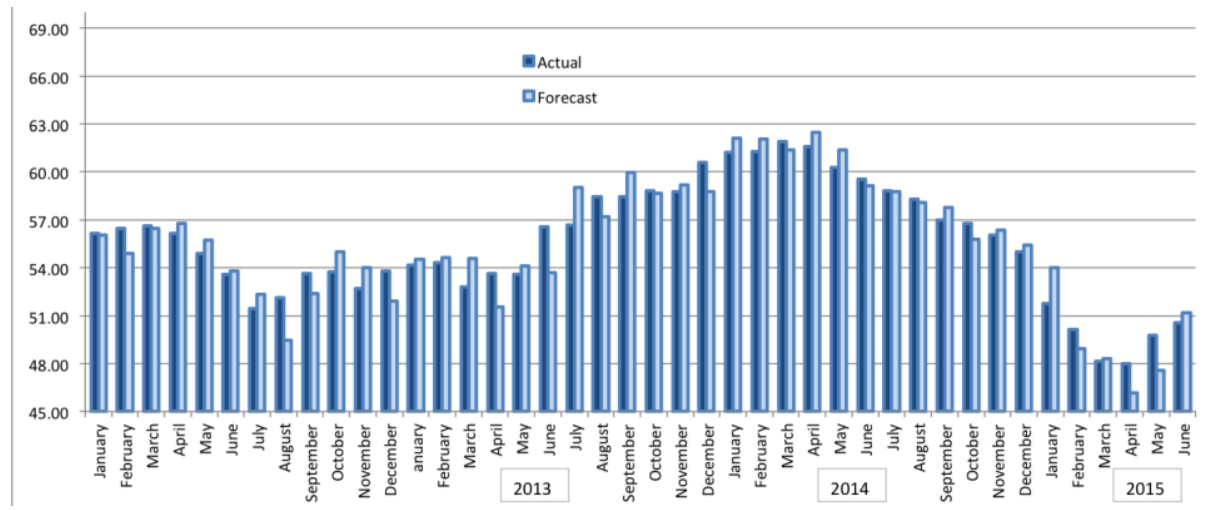

Fig. 6. Euro-peso exchange rates.

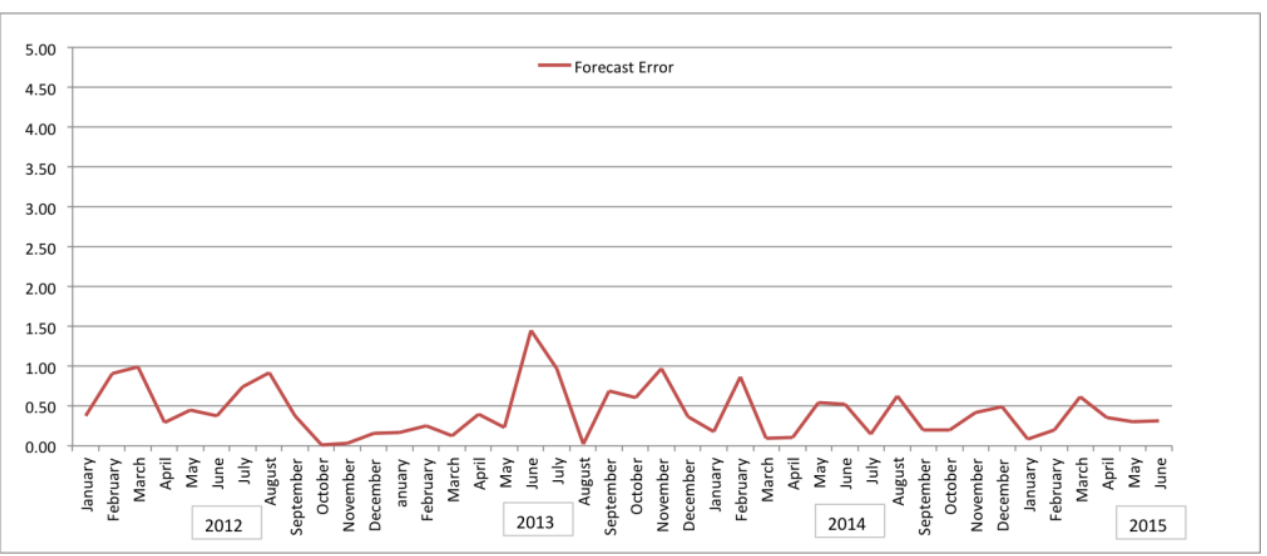

Fig. 7. Dollar-peso forecast error.

The simulations were done according to the design and methodology. With the optimized smoothing constants, Figs. 5 and 6 illustrate the actual data against the forecast values of the two different currencies 
from January 2012 to June of 2015 using Holt-Winters model.

On the other hand, the following graphs (Figs. 7 and 8) show the absolute Mean Errors (ME) of the forecasts over some period of time using the Test Set of the historical data. The forecast values are close to the actual observed data. Surprisingly, the mean forecast error for the Dollar rates is 0.43169 while the mean forecast error for Euro rates is 1.04236.

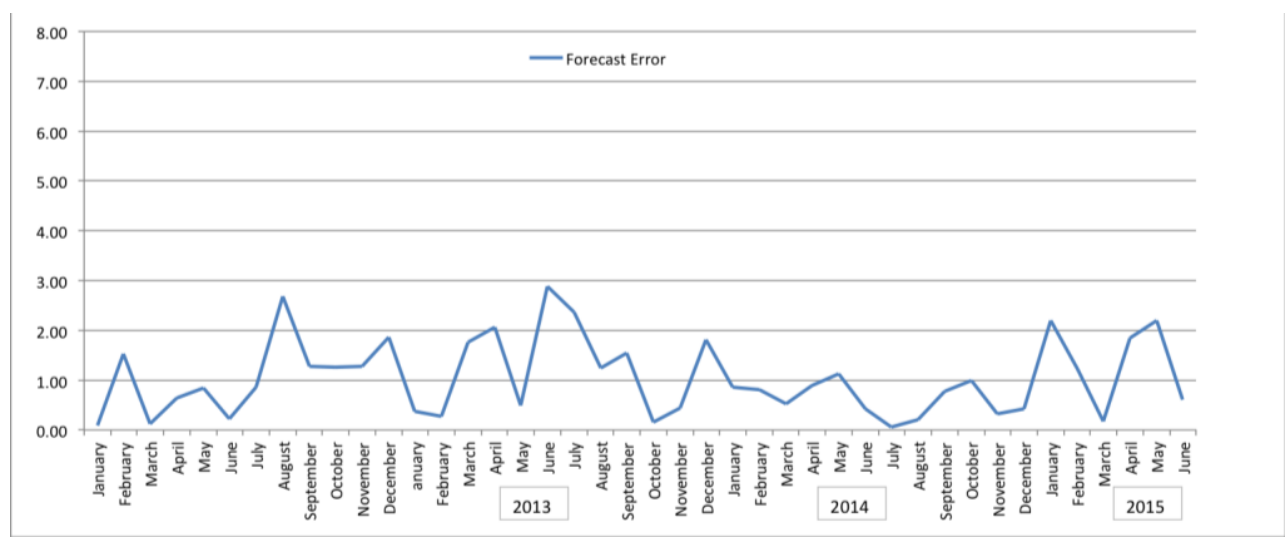

Fig. 8. Euro-peso forecast error.

\section{Conclusion}

The movements of the exchange rates are very difficult to precisely forecast. However, we have shown a technique to estimate the exchange rates with a considerable accuracy rate. Using Particle Swarm Optimization (PSO) to search for the smoothing constants of the Holt-Winters method, the built model is able to accurately forecast both the direction of the exchange rate and the actual exchange rate values for the Dollar-Peso and Euro-Peso.

Further studies can improve the estimation of the predictive power of the model with more data available. Alternatively, the technique can be applied to other exchange rate currencies in order to validate the results further. Pre-testing of the available data sets will also help determine the applicability of the Holt-Winters model assumptions and strengthen the conclusions arrived at in this study. Various other algorithms (or combinations of algorithms) can be used and benchmarked with results found here. It is also possible to extend the study by incorporating more statistical analysis with the actual data and exchange rate forecasts.

\section{Acknowledgment}

The author wishes to thank the Northwest Samar State University (NwSSU) for providing financial support in the completion of this study; and Dr. Proceso L. Fernandez of Ateneo de Manila University (AdMU) for the invaluable insights in the development of this research.

\section{References}

[1] Fu, C. (2010). Forecasting exchange rate with emd-based support vector regression. Proceedings of International Conference on Management and Service Science (pp. 1-4).

[2] Yang, H.-L., Lin, H.-C., \& Huang, S. (2011). Forecasting exchange rate using emd and bpnn optimized by particle swarm optimization. Proceedings of 3rd International Conference on Data Mining and Intelligent Information Technology Applications (pp. 294-299).

[3] Chen, S., \& Wu, J. (2000). A re-examination of purchasing power parity in japan and taiwan.

[4] Ma, Y., \& Kanas, A. (2000). Testing for a nonlinear relationship among fundamentals and exchange rates in the erm. 
[5] Bermudez, J., Segura, J., \& Vercher, E. (2007). Holt-winters forecasting: an alternative formulation applied to uk air passenger data. Journal of Applied Statistics, 34(9), 1075-1090.

[6] Maria, F., \& Eva, D. (2011). Exchange-rates forecasting: Exponential smoothing techniques and arima models. Annals of Faculty of Economics, 1(1), 499-508.

[7] Hyndman, R. S. R. J., Koehler, A. B., \& Grose, S. (2002). A state space framework automatic forecasting using exponential smoothing.

[8] Makridakis, S., \& Hibon, M. (2000). The m3-competition: results, conclusions and implications.

[9] Bermudez, J., Segura, J., \& Vercher, E. (2006). A decision support system methodology for forecasting of time series based on soft computing.

[10] Nelly, C., \& Weller. (2002). Predicting exchange rate volatility: Genetic programming versus garch and risk metrics.

[11] Andre, N., \& Leandro. (2007). Computation intelligence approaches and linear models in case studies of forecasting exchanges.

[12] Liao, J., Wang, H., \& Zhu, S. (2012). Winters-arma model based on pso and its application to opening price trend in shenzhen stock market. In X. Qu, \& Y. Yang, (Eds.), Information and Business Intelligence, volume 267 of Communications in Computer and Information Science (pp. 259-267). Springer Berlin Heidelberg.

[13] Natarajan, S., \& Periasamy. (2006). Application of particle swarm optimisation in artificial neural network for the prediction of tool life.

[14] Maria, F. C., \& Eva, D. (2011). Exchange-rates forecasting: Exponential smoothing techniques and arima models. Annals of the University of Oradea, Economic Science Series, 20(1), 499-508.

[15] Chang, J.-F., Chang, C.-W., \& Tzeng, W.-Y. (2009). Forecasting exchange rates using integration of particle swarm optimization and neural networks. Proceedings of Fourth International Conference on Innovative Computing, Information and Control (pp. 660-663).

[16] Mengliang, L., Rong, G. \& Xiuhong, W. (2008). The load forecasting using the pso-bp neural network and wavelet transform. Proceedings of 27th Chinese Control Conference (pp. 34-37).

[17] Junyou, B. (2007). Stock price forecasting using pso-trained neural networks. Proceedings of IEEE Congress on Evolutionary Computation (pp. 2879-2885).

[18]Chen, A., Hsu, Y., \& Hu, K. (2008). A hybrid forecasting model for foreign exchange rate based on a multi-neural network. Proceedings of Fourth International Conference on Natural Computation: Vol. 5 (pp. 293-298).

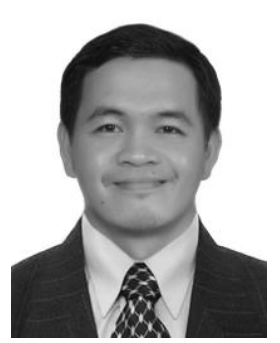

Riz Rupert L. Ortz is an assistant professor of Northwest Samar State University (NwSSU), Calbayog City, Samar, Philippines. He finished his doctorate degree (Ph.D. in computer science) in Ateneo de Manila University, Quezon City, Philippines. His research interest includes algorithm design, computer simulation, optimization and forecasting. 